\title{
Young Schema Questionnaire: Factor Structure and Specificity in Relation to Anxiety in Chinese Adolescents
}

\author{
Yulei Yan', Jianping Wang ${ }^{1,2} \bowtie$, Wei Yu' ${ }^{1}$, Li He ${ }^{1,3}$, and Tian P.S. Oei ${ }^{4,5}$ \\ ${ }^{1}$ School of Psychology, Beijing Normal University, Beijing, China \\ ${ }^{2}$ Capital Medical University, Beijing, China \\ ${ }^{3}$ Teacher's College of Beijing Union University, Beijing, China \\ ${ }^{4}$ University of Queensland, Brisbane, Australia \\ ${ }^{5}$ James Cook University, Singapore
}

Objective The goal of this study was to examine the factor structure of Young Schema Questionnaire-short form (YSQ-SF) in a sample of Chinese adolescents, and to explore which maladaptive schemas were associated with anxiety symptoms.

Methods YSQ-SF was administered to 983 nonclinical Chinese adolescents aged 13-17 years. Confirmatory factor analyses with weighted least squares means and variance adjusted estimation were conducted to examine the factor structure of YSQ-SF. Stepwise regression analyses were performed to identify schemas associated with anxiety symptoms.

Results A bifactor model with 15 correlated factors fitted the data better than other priori defined models. Stepwise regressions showed Vulnerability to harm, Abandonment, Emotional inhibition, Subjugation, and Unrelenting standards schemas were associated with anxiety symptoms. Furthermore, different anxiety symptoms were associated with common and distinctive schemas.

Conclusion Maladaptive schemas were already stably formed in Chinese adolescents, however there's no robust evidence for the existence of domain. The explanatory value of maladaptive schemas for understanding psychopathology of anxiety in adolescence is discussed.

Psychiatry Investig 2018;15(1):41-48

Key Words Young schema questionnaire, Factor structure, Schema, Adolescent, Anxiety.

\section{INTRODUCTION}

Anxiety disorder is the most common psychiatric disorder in adolescents, with a high prevalence estimate ranging from $11 \%{ }^{1}$ to $31.9 \%,{ }^{2}$ which can bring about impairment of functioning, including academic performance, self-esteem and social interactions, ${ }^{3,4}$ as well as adult mental health problems. ${ }^{5}$ Distorted cognition accounts for the dysfunctional emotions such as anxiety and depression, while maladaptive schemas underlie the development and maintenance of distorted cognitions. ${ }^{6}$ Schemas are basic beliefs about the self, others and the world, influenced by early childhood experiences with

Received: April 8, 2016 Revised: September 26, 2016

Accepted: April 17, 2017 Available online: November 21, 2017

$\square$ Correspondence: Jianping Wang

School of Psychology, Beijing Normal University, No. 19 Xinjiekouwai Street,

Haidian District, Beijing 100875, P. R. China

Tel: +86 15010037261, Fax: +86 58808187, E-mail: wjpbnu@gmail.com

(a) This is an Open Access article distributed under the terms of the Creative Commons Attribution Non-Commercial License (http://creativecommons.org/licenses/bync/4.0) which permits unrestricted non-commercial use, distribution, and reproduction in any medium, provided the original work is properly cited. primary caregivers, making people vulnerable to psychological problems. ${ }^{6}$ Therefore, the investigation of early maladaptive schemas could help understand the pathology of anxiety in adolescence.

Young extended Beck's cognitive theory by identifying 15 maladaptive schemas ${ }^{7}$ and developed Young Schema Questionnaire-Long Form (YSQ-LF) and then a short form (YSQSF). ${ }^{8}$ The reliability and validity of both measures have been widely confirmed, ${ }^{9,10}$ and YSQ-SF has proved not to be significantly different from YSQ-LF in the internal consistency, reliability or discriminant validity. ${ }^{11}$ Most studies generally have confirmed a 15 lower-order factor structure as Young proposed, ${ }^{10}$ although some findings varies slightly, such as a 13- factor solution in Korean and Australian undergraduate students samples, ${ }^{12}$ or a 14-factor solution in a Chinese undergraduate students sample. ${ }^{13}$ However, there have been hot debates on the higher-order factor structure (i.e. schema domain). Young first proposed 5 domains to classify 15 schemas, ${ }^{7}$ however, while other studies examining the higher-order factor structure of YSQ-SF, the numbers of domains were 
always inconsistent. Some studies using exploratory factor analysis revealed 3 or 4 -domian solution, ${ }^{9,14}$ while other studies using confirmatory factor analysis showed that all models including 3, 4 and 5-domain solutions seemed parallel. ${ }^{15}$ Given that previous findings are mixed, Kriston et al. ${ }^{16}$ claimed that the higher-order structure might not exist, instead, the structure could be represented by a bifactor model including correlated first-order specific schema factors and a first-order generic factor (Figure 1). Specifically, the specific factors correspond to 15 schemas proposed by Young, while the generic factor could be a relatively stable trait that influences the development of pathology such as neuroticism or negative affectivity, ${ }^{16}$ or negative self-image. ${ }^{17}$ Given the fact that many schemas are overlapped theoretically and the domain seems to be an artifical concept to cluster the schemas, more researches are needed to examine whether domain exist.

Furthermore, maladaptive schemas originate early in life, and might still be in the process of forming during childhood and adolescence, so the structure of schemas might be less diversified in a youth sample than in an adult sample. ${ }^{18}$ However, literatures on maladaptive schemas in youth are too limited $^{15,18,19}$ to draw a conclusion whether maladaptive schemas

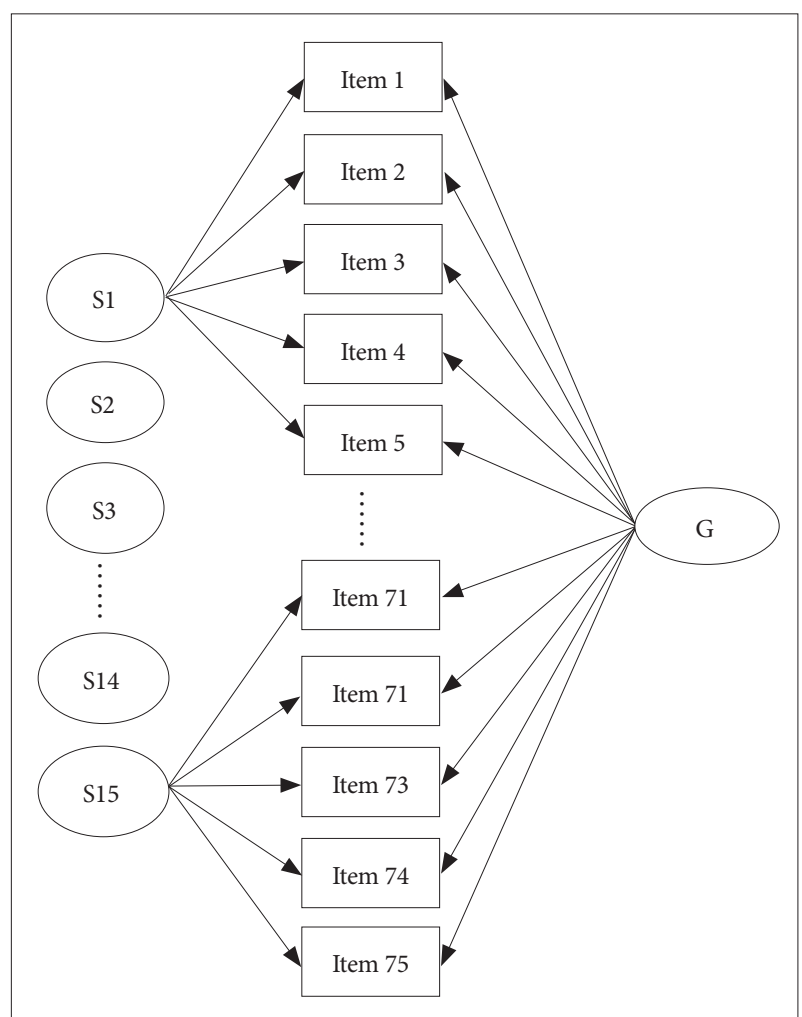

Figure 1. Bifactor model of YSQ-SF. S: schema factors, G: firstorder generic factor. The paths betwen schema and items were consistent with the model proposed by Young, which were not displayed completely for the visual simplicity. All schema factors were correlated in this bifactor model. Also in the interest of visual simplicity, the paths between schema factors were not displayed. The generic factor was not correlated with any schema factors. have already formed stably in adolescents.

The Beck's cognitive content specificity hypothesis advocated that each type of pathology was associated with a unique set of schemas. ${ }^{20}$ Regarding the anxiety symptoms, many studies have confirmed that Disconnection and rejection and Impaired autonomy domains were significantly related to anxiety symptoms. ${ }^{18,21-23}$ However, whether domains exist still need exploration. Moreover, some schemas which belong to these domains are not related to anxiety symptoms. For example, Dependence and Failure to achieve schemas belong to Impaired autonomy domain, but are related to depression symptoms ${ }^{15}$ rather than anxiety symptoms. ${ }^{15,24}$ Given that, it would be helpful to examine schemas rather than schema domains as potential predictors of anxiety symptoms, in order to achieve a more precise understanding of adolescent anxiety.

However, previous findings regarding the schemas related to anxiety symptoms are inconsistent. ${ }^{25}$ Also, the studies examining schemas related to adolescent anxiety reported inconsistent findings. Muris conducted a modified version of YSQ in a sample of non-clinical adolescents, and reported that anxiety symptoms were uniquely predicted by by Emotional inhibition, Abandonment and Social isolation schemas. ${ }^{18}$ Van Vlierberghe et al. ${ }^{15}$ employed structured diagnostic interview and found Abandonment, Failure to achieve, Dependence/incompetence, Unrelenting standards, and Entitlement/grandiosity schemas were positively predictive for the presence of an anxiety disorder in youth. A recent study using Malaysian nonclinical adolescents sample showed that adolescent anxiety were associated with Vulnerability to harm, Abandonment, Pessimism/worry and Enmeshment schemas. ${ }^{24}$

These inconsistencies may reflect methodological differences between studies, such as different measurements and samples (e.g. clinical versus non-clinical sample, adults versus youth, and the group under different cultural context). Moreover, the schemas are highly correlated with each other, complicating the evaluation of relative importance of each schema to particular pathology. Therefore Van Vlierberghe et al. ${ }^{15}$ suggested some statistic techniques such as regression analysis should be used to control overlapping schemas.

\section{The current study}

The current study examined the lower and higher-order factor structure of YSQ-SF, and investigated the association between maladaptive schemas and anxiety symptoms in Chinese adolescents. According to theoretical considerations, the specific research questions were: 1) whether domain (higher-order structure) exists? It was expected that domain doesn't exist; instead, a bifactor model (Figure 1) might better represent the factor structure of YSQ-SF, 2) which maladaptive schemas were associated with anxiety symptoms in Chinese adoles- 
cents? Given that previous findings were quite inconsistent, no specific assumptions would be made.

\section{METHODS}

\section{Participants}

Participants included 983 adolescents (525 females and 458 males) with a mean age of 15.44 years $\left(\mathrm{SD}=0.98\right.$; range $13^{-}$ 17). Ninety percent $(\mathrm{n}=884)$ of participants lived with both parents, $7.4 \%(n=73)$ lived with single parent who were divorced or separated, and $2.6 \%(\mathrm{n}=26)$ haven't reported their parents' marital status. Only 73 of the whole sample (7.26\%) have siblings in their family. Most adolescents reported their parents graduated from a university (father, $\mathrm{n}=797,79.30 \%$; mother, $n=773,76.91 \%)$. Participants were drawn from four secondary schools.

\section{Measures}

The Young Schema Questionnaire-Short Form (YSQ-SF) is a 75-item self-report questionnaire that measures 15 EMS (early maladaptive schemas). ${ }^{7}$ EMS are grouped in five broad domains: Disconnection and rejection, Impaired autonomy, Impaired limits, Other-directedness, and Over vigilance and inhibition. Each item is rated on a Likert scale from 1 (completely untrue of me) to 6 (describes me perfectly). The YSQSF shows adequate reliability and validity in predicting psychopathology in different studies. ${ }^{9.26}$ The Chinese Version of YSQ-SF has good psychometric properties in undergraduate students, but only verified 14 factors and 3 domains. ${ }^{13}$

The Screen for Child Anxiety Related Emotional Disorders (SCARED) is a 41-item self-report measure designed to screen for DSM-IV anxiety disorders. ${ }^{27}$ The SCARED includes 5 factors: panic, generalized anxiety, separation anxiety, social phobia, and school phobia. Each item is rated on a Likert scale from 0 (completely untrue of me) to 2 (true or often true). The Chinese Version of SCARED has good psychometric properties. ${ }^{28}$ Given school phobia is not an anxiety symptom according to DSM-IV-TR, ${ }^{29}$ it was not examined in the current study.

\section{Procedure}

The University Institutional Review Board approved this study. This study was part of a larger research project on schoolbased intervention for anxious adolescents. Nine secondary schools in Beijing were contacted to take part in this intervention project and 4 schools agreed. This relatively low rate of agreement was largely due to their concern with the time incompatibility between intervention and school schedule. Participants were comprised of students who were in grade 7,8 , 9,10 , and 11 , whereas students who were in grade 12 were not included in this project, because the schools didn't want anything to interrupt their study.

After obtaining written consent from parents and adolescents, students completed a battery of questionnaires. Since the written consent was sent to parents and students after a psychological education lecture about adolescent anxiety disorders, no parents objected to the participation of survey, while one adolescent refused to attend. Participants completed the questionnaires during classes, while one research assistant was present to make sure the adolescents received standard instructions.

The Chinese version of the YSQ-SF was obtained from Cui et al. ${ }^{13}$ who use undergraduate students as the sample. Considering the Chinese version of YSQ-SF which only includes 14 factors might not represent the factor structure in Chinese adolescents, we obtained the complete translated version including 75 items and modified some items to adjust to adolescents' life situation. For example, item 41: "I have not been able to separate myself from my parent(s) the way other people my age seem to." Since adolescents couldn't separate themselves from parents completely, we modified it into "I have not been able to keep my own independence in my family the way other people my age seem to." We've modified item 41 and 45 . The modified questionnaire was tested in a pilot study on 30 adolescents, and the content of items was revised if adolescents couldn't perceive the correct meanings.

\section{Statistical analyses}

Cases with more than $10 \%$ missing values on the YSQ-SF $(n=22)$ were removed. Then the missing values for the YSQ$\mathrm{SF}$ were imputed using the expectation maximization (EM) algorithm. ${ }^{30}$ YSQ-SF has ordered categorical indicators and do not follow a multivariate normal distribution. Muthén introduced the weighted least squares means and variance adjusted estimation (WLSMV) for analyzing ordinal data, ${ }^{31}$ thus Mplus $7.11^{32}$ was used to run confirmatory factor analysis (CFA) with WLSMV for order categorical indicators, in order to assess the fit of several a priori defined models, including a 15 -factor model proposed by Young, ${ }^{7}$ a 13 -factor model, ${ }^{12}$ a 14 -factor model, ${ }^{13}$ a 5 -domain solution, ${ }^{7}$ a 4 -domain solution, ${ }^{14,17}$ a 3-domain solution 13 and bifactor models with and without correlations between the first-order schema factors. ${ }^{16}$

The chi-square goodness-of-fit statistic divided by its degrees of freedom $\left(\chi^{2} / \mathrm{df}\right)$, the Robust Comparative Fit Index (CFI), the Tucker-Lewis Index (TLI), and the root mean square error of approximation (RMSEA) were used as goodness of fit indices. Ratios of $\left(\chi^{2} / \mathrm{df}\right)$ ranged from 2:1-5:1 indicates an acceptable fit. ${ }^{33}$ The values of CFI and TLI greater than 0.90 indicates a good model fit. ${ }^{33}$ RMSEA less than 0.08 and 0.06 represents respectively, an acceptable and good model fit. ${ }^{34}$ 
Regarding to the association between schemas and anxiety symptoms, potential covariables such as age and gender were examined. Pearson correlation analysis was conducted to explore the association between age and anxiety symptoms. Oneway ANOVA was conducted to explore whether gender had influence on anxiety symptoms. Furthermore, a series of stepwise regressions was then carried out to examine the most parsimonious set of schemas as predictors of various anxiety symptoms, with the measure of anxiety and its subscales as the dependent variables separately, 15 schemas as the independent variables, and the potential covariables also entered into the regression analysis. These statistical analyses were performed with SPSS 20.0 (IBM Corp., Armonk, NY, USA).

\section{RESULTS}

\section{CFA of priori defined models}

On the scale of YSQ-SF from 1 to 6 , the means of the 75 items ranged from 1.37 to 3.93 with a median of 1.94. Skewness and kurtosis values ranged from -0.31 to 3.24 and -1.19 to 11.42 with medians of 1.56 and 1.38 , respectively. A small proportion $(0.51 \%)$ of the Pearson product-moment correlations of 75 items exceeded 0.70 . The median correlation between items was 0.26 . The fit indices of all priori defined models are presented in Table 1. As can be seen from Table 1, Young's theoretical model (M1) with 15 schema factors without higherorder domains exhibited a good fit (CFI and TLI $\geq 0.90, \chi^{2} /$ $\mathrm{df}<3$, RMSEA $<0.06$ ), while either M2 or M3 didn't reach threshold for acceptable models. In addition, M1 exhibited good internal consistency. As can be seen from Table 2, the factor reliability coefficients ranged from 0.76 for "Entitlement/ Grandiosity" to 0.92 for "Failure to achieve" with a median of 0.84 .

All the higher-order factor models (M4, M5, and M6) produced comparable estimates and exhibited good fits. Two bifactor models (M7 and M8) also resulted in good fits. However, M8 showed the best fit (CFI and TLI $=0.96, \chi^{2} / \mathrm{df}<3, \mathrm{RMSEA}=$
0.04) among all the models tested.

In summary, Young's theoretical 15-schema model fitted the data better than all other lower-order factor models, whilst all the higher-order domain models paralleled, but the bifactor model with correlated schemas resulted in the best fit.

\section{Maladaptive schemas associated with anxiety symptoms}

With respect to the potential covariables, Pearson correlation analysis showed the scores of SCARED and all the subscales were all significantly related to the participant's age (SCARED, $r=-0.497, \mathrm{p}<0.001$; all subscales, nps $<0.001$ ). The result of Pearson correlation analysis can be seen in Table 3. The result of ANOVA showed the gender didn't have significant effects on the subscale of anxiety symptoms (nps $>0.05$ ). Therefore only age entered into the next analysis as covariable.

A series of stepwise regressions were conducted to assess

Table 2. Internal consistency for the subscales of $\mathrm{YSQ}-\mathrm{SF}(\mathrm{N}=983)$

\begin{tabular}{lc}
\hline \multicolumn{1}{c}{ Schema } & Cronbach's alpha \\
\hline 1. Emotional deprivation & 0.87 \\
2. Abandonment & 0.82 \\
3. Mistrust/abuse & 0.90 \\
4. Social isolation/alienation & 0.87 \\
5. Defectiveness/shame & 0.84 \\
6. Failure & 0.92 \\
7. Dependence/incompetence & 0.81 \\
8. Vulnerability to harm or illness & 0.84 \\
9. Enmeshment/undeveloped self & 0.81 \\
10. Subjugation & 0.79 \\
11. Self-sacrifice & 0.82 \\
12. Emotional inhibition & 0.87 \\
13. Unrelenting standards & 0.80 \\
14. Entitlement/grandiosity & 0.81 \\
15. Insufficient self-control/self-discipline & 0.82
\end{tabular}

YSQ-LF: Young Schema Questionnaire-Long Form

Table 1. Summary of fit indices of a priori defined models $(\mathrm{N}=983)$

\begin{tabular}{|c|c|c|c|c|c|c|}
\hline Model & $\chi^{2}$ & df & $\chi^{2} / \mathrm{df}$ & CFI & TLI & RMSEA \\
\hline M1 15 correlated factors $^{7}$ & 7124.35 & 2595 & 2.75 & 0.94 & 0.93 & 0.04 \\
\hline M2 13 correlated factors ${ }^{12}$ & 43545.67 & 2633 & 16.54 & 0.45 & 0.42 & 0.13 \\
\hline M3 14 correlated factors $^{13}$ & 46584.83 & 2620 & 17.78 & 0.40 & 0.37 & 0.13 \\
\hline M4 5 domains $^{7}$ & 9520.69 & 2675 & 3.56 & 0.91 & 0.90 & 0.05 \\
\hline M5 4 domains $^{14}$ & 9531.00 & 2679 & 3.56 & 0.91 & 0.90 & 0.05 \\
\hline M6 3 domains ${ }^{13}$ & 9548.90 & 2680 & 3.56 & 0.91 & 0.90 & 0.05 \\
\hline M7 uncorrelated bifactor model ${ }^{16}$ & 9127.02 & 2625 & 3.48 & 0.92 & 0.92 & 0.05 \\
\hline M8 correlated bifactor model ${ }^{16}$ & 5841.14 & 2520 & 2.32 & 0.96 & 0.96 & 0.04 \\
\hline
\end{tabular}

CFI: Robust Comparative Fit Index, TLI: Tucker-Lewis Index, RMSEA: root mean squared error of approximation 
Table 3. Pearson correlations among age and anxiety symptoms $(\mathrm{N}=983)$

\begin{tabular}{lcccccc}
\hline \multicolumn{1}{c}{ Variable } & 1 & 2 & 3 & 4 & 5 & 6 \\
\hline 1. Age & - & & & & \\
2. PA & $-0.49^{* * *}$ & - & & & \\
3. GA & $-0.37^{* * *}$ & $0.79^{* * *}$ & - & & \\
4. SE & $-0.52^{* * *}$ & $0.80^{* * *}$ & $0.74^{* * *}$ & - & & \\
5. SO & $-0.36^{* * *}$ & $0.65^{* * *}$ & $0.70^{* * *}$ & $0.64^{* * *}$ & - & - \\
6. SC & $-0.43^{* * *}$ & $0.69^{* * *}$ & $0.63^{* * *}$ & $0.67^{* * *}$ & $0.53^{* * *}$ & $0.77^{* * *}$ \\
7. SCARED & $-0.50^{* * *}$ & $0.93^{* * *}$ & $0.91^{* * *}$ & $0.89^{* * *}$ & $0.81^{* * *}$ & - \\
\hline
\end{tabular}

${ }^{* * *} \mathrm{p}<0.001$. SCARED: total score of the Screen for Child Anxiety Related Emotional Disorders, PA: somatic/panic, GA: generalized anxiety, SE: separation anxiety, SO: social phobia, SC: school phobia

Table 4. The result of stepwise regression analysis $(\mathrm{N}=983)$

\begin{tabular}{|c|c|c|c|c|c|c|c|c|c|c|}
\hline \multirow{2}{*}{ Schemas } & \multicolumn{2}{|c|}{ SCARED } & \multicolumn{2}{|c|}{ PA } & \multicolumn{2}{|c|}{ GA } & \multicolumn{2}{|c|}{ SE } & \multicolumn{2}{|c|}{$\mathrm{SO}$} \\
\hline & $\beta$ & $\mathrm{t}$ & $\beta$ & $\mathrm{t}$ & $\beta$ & $\mathrm{t}$ & $\beta$ & $\mathrm{t}$ & $\beta$ & $\mathrm{t}$ \\
\hline $\mathrm{AB}$ & 0.25 & $9.32^{* * *}$ & 0.21 & $7.50^{* * *}$ & 0.25 & $9.21^{* * *}$ & 0.23 & $8.03^{* * *}$ & 0.22 & $7.38^{* * *}$ \\
\hline $\mathrm{VH}$ & 0.21 & $7.12^{* * *}$ & 0.23 & $7.34^{* * *}$ & 0.25 & $8.15^{* * *}$ & 0.24 & $8.43^{* * *}$ & - & - \\
\hline SB & 0.13 & $4.39^{* * *}$ & 0.14 & $4.49^{* * *}$ & 0.14 & $4.67^{* * *}$ & - & - & 0.13 & $3.92^{* * *}$ \\
\hline EI & 0.14 & $5.24^{* * *}$ & - & - & 0.16 & $6.13^{* * *}$ & - & - & 0.31 & $9.88^{* * *}$ \\
\hline US & -0.05 & $-2.29^{*}$ & - & - & - & - & - & - & -0.09 & $-3.33^{* *}$ \\
\hline
\end{tabular}

${ }^{*} \mathrm{p}<0.05,{ }^{* *} \mathrm{p}<0.01,{ }^{* * *} \mathrm{p}<0.001$. SCARED: total score of the Screen for Child Anxiety Related Emotional Disorders, PA: somatic/panic, GA: generalized anxiety, SE: separation anxiety, SO: social phobia, AB: abandonment, VH: vulnerability to harm or illness, EI: emotional inhibition, SB: subjugation, US: unrelenting standards

the association between schemas and anxiety symptoms with age as a covariable. As Table 4 showed, different anxiety symptoms can be predicted by common and distinctive schemas. The subscales of Vulnerability to harm, Abandonment, Emotional inhibition, Subjugation and Unrelenting standards contributed significantly to the prediction of anxiety symptoms. Regarding SCARED scores, $30.7 \%$ of the variance was accounted for by those identified schemas, while $24.7 \%$ of the variance was accounted for by age. Panic symptom was significantly predicted by Vulnerability to harm, Abandonment and Subjugation schemas $\left(\mathrm{R}^{2}=0.23\right)$; similarly, general anxiety was significantly predicted by Vulnerability to harm, Abandonment, Emotional inhibition and Subjugation schemas $\left(\mathrm{R}^{2}=0.39\right)$; furthermore, separation anxiety was significantly predicted by Vulnerability to harm and Abandonment schemas $\left(\mathrm{R}^{2}=0.17\right)$; and social phobia was significantly predicted by Emotional inhibition, Abandonment, Subjugation and Unrelenting standards schemas $\left(\mathrm{R}^{2}=0.24\right)$.

\section{DISCUSSION}

\section{Factor structures of YSQ-SF}

Results of this study provide the evidence that Young's theoretical 15-schema model is the best lower-order factor model, which parallels other researches using Western adolescent sample ${ }^{15}$ or adult sample. ${ }^{16}$ This result contradicted with pre- vious reports suggesting a 13-schema factor model derived from an Asian sample ${ }^{12}$ or a 14-schema factor model derived from Chinese undergraduate student, ${ }^{13}$ probably implying that cultural context doesn't have significant influence on the factor structure of YSQ-SF. In addition, the schemas in Chinese adolescents seem diversified enough, which is contradicted with the hypothesis that maladaptive schemas might still be in the process of forming during adolescence. ${ }^{18}$

Moreover, it should be noted that when examining the factor structure of a questionnaire, exploratory factor analysis (EFA) always resulted in solutions that adequately fit specific data but could not be reproducible in other settings, which could be a reason for the variety and inconsistency of prior defined models. Some researchers warned that EFA might cause the "overfitting" of the resulting models and the generalizability might be overemphasized. ${ }^{16}$ In compliance with this perspective, the finding of this study showed that 14-schema model in Chinese Version of YSQ-SF ${ }^{13}$ which was derived from EFA failed to reach an acceptable fit; instead, 15-schema model proposed by Young shows a good fit.

With respect to the higher-order factor structure, there's no robust evidence that domains exist, given that bifactor model fitted the data best. This finding was in line with Kriston et al. ${ }^{16}$ findings which reported that a bifactor model with correlated first-order schema overrode other prior defined models. Hoffart et al. ${ }^{17}$ also reported that several higher-or- 
der models derived empirically had only minor differences, and the highly correlated schemas and domains suggested that a common factor existed across the schemas. This common factor seems to be a relatively stable trait that influences the development of pathology. Some researchers named this common factor as neuroticism or negative affectivity ${ }^{16}$ or negative self-image. ${ }^{17}$ Although the nature of this common factor still needs further exploration, it seems that domain might not be a valid concept.

\section{Maladaptive schemas related to anxiety symptoms}

The current study showed that anxiety symptoms in Chinese adolescents could be predicted by Vulnerability to harm, Abandonment, Emotional inhibition, Subjugation and Unrelenting standards schemas. These identified schemas were partly in line with previous findings regarding adolescent anxiety respectively, ${ }^{15,18,24}$ Muris found that adolescent anxiety symptoms were uniquely predicted by by Emotional inhibition, Abandonment and Social isolation schemas. ${ }^{18}$ van Vlierberghe et al. ${ }^{15}$ found Abandonment, Failure to achieve, Dependence/incompetence, Unrelenting standards, and Entitlement/grandiosity schemas were positively predictive for the presence of an anxiety disorder. A recent study using Malaysian nonclinical adolescents sample showed that adolescent anxiety could be predicted by Vulnerability to harm, Abandonment, Pessimism/worry and Enmeshment schemas. ${ }^{24}$

Abovementioned evidences indicated Abandonment schema was the most common predictor of adolescent anxiety in different studies. Also, our findings revealed Abandonment schema was associated with every subscale of SCARED. It seems Abandonment schema play very significant role in the development and maintenance of anxiety. Other studies employing adult samples also revealed the strong association between Abandonment schema and anxiety symptoms. ${ }^{26,35}$ According to attachment theory, ${ }^{36}$ perceived abandonment can provoke sense of hopelessness and destroy the sense of security, resulting in higher level of anxiety symptoms. ${ }^{37}$

Although Beck proposed that vulnerability to harm is the core feature of anxiety disorders, ${ }^{38}$ the empirical studies showed inconsistent evidence. Vulnerability to harm schema unstably emerged as a predictor of anxiety symptoms in clinical or non-referred adult samples. ${ }^{25}$ Regarding the schemas related to adolescent anxiety, apart from our findings, only one previous study has confirmed Vulnerability to harm schema was strongly associated with adolescent anxiety. ${ }^{24}$ Some researchers pointed out the inconsistence of findings may be due to the inconsistence of sample group, measurement and statistic methodology. ${ }^{15}$ Besides that, we assume cultural issue may also be involved to account for the inconsistence of previous findings. Different cultural context has proved to produce sig- nificantly different schemas scores across samples. ${ }^{12}$ However, given that empirical studies available regarding the association between schemas and adolescent anxiety is quite scarce, the role of culture in this association needs further exploration.

It also can be seen from current findings that different anxiety symptom subscales was explained by overlapping groups of maladaptive schemas, indicating different anxiety symptoms are associated with common and distinctive schemas. Separation anxiety, generalized anxiety and panic symptoms share common predictors including Abandonment and Vulnerability to harm schemas. Besides the common schemas, panic symptoms also can be explained by Subjugation schema, and generalized anxiety by Subjugation and Emotional inhibition schemas, respectively. Social phobia seems to be more distinctive compared to other anxiety symptoms, since it cannot be explained by Vulnerability to harm schema which was the common factor of any other anxiety symptoms, and Unrelenting standards schema as a distinctive predictor of social phobia, was not related to any other anxiety symptoms.

This finding can help understand the diversity of potential risk factors of various anxiety symptoms. For example, social phobia and generalized anxiety are predicted by Emotional inhibition schema, which may suggest that inhibited temperament could be a risk factor for an adolescent to develop social or generalized anxiety, rather than panic or separation anxiety, which was in line with previous findings showing strong association between social anxiety and inhibition..$^{39,40}$ Through identifying the distinctive predictors of different anxiety symptoms, intervention can target the identified maladaptive schemas in order to facilitate the efficacy.

Moreover, the current study showed that Unrelenting standards schema was the only one of the predictors correlated negatively with social phobia and overall anxiety symptoms scores, implying individuals with higher standards may have lower level of social anxiety. Previous findings also reported that patients with social phobia scored lower than non-clinical population on Unrelenting standards schema. ${ }^{41}$ Elevated standards may indicate the higher level of mastery and competence, which prevent the development of social anxiety.

In addition, the current study demonstrated that the severity of anxiety symptoms decreased with greater age, which is partially in compliance with previous research results which showed most anxiety symptoms had a slight decrease in their severity over the course of 5 years. ${ }^{42}$ However, only a few schemas were significantly correlated with the participant's age, implying that schema is a relatively more stable structure as compared with symptoms, keeping in line with Young's proposition that maladaptive schemas develop early in life and serve as stable, overgeneralized belief structures. ${ }^{7,43}$ 


\section{Limitations}

The current study has several limitations that highlight the need for more research. First, the cross-sectional nature of this study does not allow any conclusions to be drawn about causeeffect relations among the assessed variables. Further research should collect longitudinal data to examine the contribution of maladaptive schemas to the development and maintaining of anxiety symptoms. Second, the study merely relied on adolescents' self-reports. Further research should use more measures (clinical interview) and multi-sources of information (parent-report and teacher-report) to confirm the results. However, it is also important to note that self-reports are probably the most effective method to assess anxiety symptoms during adolescence, because youth have been shown to be more accurate sources of information than parents and teachers regarding information about their inner states. ${ }^{44}$ Third, the sample was non-clinical, thus the results of this study could not be applicable to clinical sample. Since clinical and nonclinical studies complement each other, replication in clinical samples is required in further research. Fourth and finally, given that the study was part of school-based intervention research, the current sample did not cover a wide age range of adolescents, which might limit the generalizability of the findings.

\section{Conclusions}

The finding of this study showed that 15-schema model proposed by Young and a bifactor model with correlated specific schemas fitted better than any other prior defined models. The concept of domain seems not good enough to profile the relationship among various schemas, while the nature of common factor in the bifactor model need more exploration in future research. In addition, the YSQ-SF showed consistent factor structure and good internal consistency in Chinese adolescents, indicating that YSQ-SF was a valid instrument to access the maladaptive schemas in such population.

This study also provided evidence that Vulnerability to Harm, Abandonment, Emotional Inhibition, Subjugation and Unrelenting Standards schemas have strong association with anxiety symptoms, keeping in line with Beck's theory that deeper schemas might increase the risk of developing psychopathology. ${ }^{45}$

\section{REFERENCES}

1. Costello EJ, Egger HL, Copeland W, Erkanli A, Angold A. The Developmental Epidemiology of Anxiety Disorders: Phenomenology, Prevalence, and Comorbidity. In: Silverman WK, Field AP, Editors. Anxiety Disorders in Children and Adolescents: Research, Assessment and Intervention. Cambridge: Cambridge University Press, 2011, p.631-648.

2. Merikangas KR, He JP, Burstein M, Swanson SA, Avenevoli S, Cui L, et al. Lifetime prevalence of mental disorders in US adolescents: results from the National Comorbidity Survey Replication-Adolescent Supplement (NCS-A). J Am Acad Child Adolesc Psychiatry 2010;49:980-
989.

3. McLoone J, Hudson JL, Rapee RM. Treating anxiety disorders in a school setting. Educ Treat Child 2006;29:219-242.

4. Barrett PM, Pahl KM. School-based intervention: examining a universal approach to anxiety management. Aust J Guid Couns 2006;16:5575.

5. Dadds MR, Spence SH, Holland DE, Barrett PM, Laurens KR. Prevention and early intervention for anxiety disorders: a controlled trial. J Consult Clin Psychol 1997;65:627-635.

6. Beck JS. Cognitive Therapy: Basics and Beyond. New York: Guilford Press; 1995.

7. Young JE, Klosko JS, Weishaar ME. Schema Therapy: a Practitioner's Guide. New York: Guilford Press; 2003.

8. Young JE. Cognitive Therapy for Personality Sisorders: A Schema-Focused Approach (Rev). Sarasota, FL: Professional Resource Exchange; 1994.

9. Calvete E, Estévez A, López de Arroyabe E, Ruiz P. The schema questionnaire--short form: structure and relationship with automatic thoughts and symptoms of affective disorders. Eur J Psychol Assess 2005;21:9099.

10. Oei TP, Baranoff J. Young schema questionnaire: review of psychometric and measurement issues. Aust J Psychol 2007;59:78-86.

11. Stopa L, Thorne P, Waters A, Preston J. Are the short and long forms of the Young Schema Questionnaire comparable and how well does each version predict psychopathology scores? J Cogn Psychother 2001;15: 253-272.

12. Baranoff J, Oei TP, Cho SH, Kwon SM. Factor structure and internal consistency of the Young Schema Questionnaire (Short Form) in Korean and Australian samples. J Affect Disord 2006;93:133-140.

13. Cui L, Lin W, Oei TP. Factor structure and psychometric properties of the young schema questionnaire (short form) in chinese undergraduate students. Int J Mental Health Addict 2011;9:645-655.

14. Lee CW, Taylor G, Dunn J. Factor structure of the schema questionnaire in a large clinical sample. Cogn Ther Res 1999;23:441-451.

15. Van Vlierberghe L, Braet C, Bosmans G, Rosseel Y, Bögels S. Maladaptive schemas and psychopathology in adolescence: on the utility of Young's schema theory in youth. Cogn Ther Res 2010;34:316-332.

16. Kriston L, Schäfer J, von Wolff A, Härter M, Hölzel LP. The latent factor structure of Young's early maladaptive schemas: are schemas organized into domains? J Clin Psychol 2012;68:684-698.

17. Hoffart A, Sexton H, Hedley LM, Wang CE, Holthe H, Haugum JA, et al. The structure of maladaptive schemas: a confirmatory factor analysis and a psychometric evaluation of factor-derived scales. Cogn Ther Res 2005;29:627-644.

18. Muris P. Maladaptive schemas in non-clinical adolescents: relations to perceived parental rearing behaviours, big five personality factors and psychopathological symptoms. Clin Psychol Psychother 2006;13:405413.

19. Saritas D, Gençöz T. Psychometric properties of "young schema questionnaire-short form 3" in a turkish adolescent sample. J Cogn Behav Psychother 2011;11:83-96.

20. Baranoff J, Oei TPS. The Cognitive Content-Specificity Hypothesis: Contribution to Diagnosis and Assessment. In: Brown G, Clark D, Editors. Assessment in Cognitive Therapy. New York: Guilford Press, 2014, p.172-196.

21. Creveling CC. The Role of Parental Control, Youth's Cognitive Schemas and Emotion Regulation Skills on Youth Anxiety across Different Age Groups. New Orleans: Tulan University; 2012.

22. Orue I, Calvete E, Padilla P. Brooding rumination as a mediator in the relation between early maladaptive schemas and symptoms of depression and social anxiety in adolescents. J Adolesc 2014;37:1281-1291.

23. Calvete E, Orue I, Hankin BL. A longitudinal test of the vulnerabilitystress model with early maladaptive schemas for depressive and social anxiety symptoms in adolescents. J Psychopathol Behav Assess 2015; 37:85-99. 
24. Mousavi SE, Low WY, Hashim AH. The relationships between perceived parental rearing style and anxiety symptoms in Malaysian adolescents: the mediating role of early maladaptive schemas. J Depress Anxiety 2016 In press.

25. Hawke LD, Provencher MD. Schema theory and schema therapy in mood and anxiety disorders: a review. J Cogn Psychother 2011;25:257276 .

26. Schmidt NB, Joiner Jr TE, Young JE, Telch MJ. The schema questionnaire: investigation of psychometric properties and the hierarchical structure of a measure of maladaptive schemas. Cogn Ther Res 1995;19: 295-321.

27. Birmaher B, Brent DA, Chiappetta L, Bridge J, Monga S, Baugher M. Psychometric properties of the Screen for Child Anxiety Related Emotional Disorders (SCARED): a replication study. J Am Acad Child Adolesc Psychiatry 1999;38:1230-1236.

28. Su L, Wang K, Fan F, Su Y, Gao X. Reliability and validity of the screen for child anxiety related emotional disorders (SCARED) in Chinese children. J Anxiety Disord 2008;22:612-621.

29. APA. Diagnostic and Statistical Manual-Text Revision (DSM-IV-TRim, 2000). Washington, DC: American Psychiatric Association; 2000.

30. G Domadia S, Ardeshana MA, Bhatt SD. Advances in Em (Expectation-Maximization) algorithm for image classification as per cost and accuracy. IJRCCT 2014;3: 65-69.

31. Muthen B. Goodness of Fit with Categorical and Other Nonnormal Variables. In: Bollen K, Long J, Editors. Testing Structural Equation Models. Newbury Park, CA: SAGE focus Editions, 1993, p.111-135.

32. Muthén L, Muthén B. Mplus: Statistical Analysis with Latent Variables. User's Guide (Version 7.11). Los Angeles, CA: Muthen and Muthen; 2013.

33. Hu Lt, Bentler PM. Cutoff criteria for fit indexes in covariance structure analysis: Conventional criteria versus new alternatives. Struct Equ Modeling 1999;6:1-55.

34. Browne MW, Cudeck R. Alternative ways of assessing model fit. Sociol Methods Res 1992;21:230-258.

35. Welburn K, Coristine M, Dagg P, Pontefract A, Jordan S. The Schema
Questionnaire-Short Form: factor analysis and relationship between schemas and symptoms. Cogn Ther Res 2002;26:519-530.

36. Bowlby J. Separation: anxiety and Anger. Attachment and Loss. New York: Basic Books; 1973.

37. Bögels SM, Brechman-Toussaint ML. Family issues in child anxiety: attachment, family functioning, parental rearing and beliefs. Clin Psychol Rev 2006;26:834-856.

38. Beck AT, Emery G, Greenberg RL. Anxiety Disorders and Phobias: A Cognitive Perspective. New York: Basic Books; 2005.

39. Schwartz CE, Snidman N, Kagan J. Adolescent social anxiety as an outcome of inhibited temperament in childhood. J Am Acad Child Adolesc Psychiatry 1999;38:1008-1015.

40. Biederman J, Hirshfeld-Becker DR, Rosenbaum JF, Hérot C, Friedman D, Snidman N, et al. Further evidence of association between behavioral inhibition and social anxiety in children. Am J Psychiatry 2001;158: 1673-1679.

41. Pinto-Gouveia J, Castilho P, Galhardo A, Cunha M. Early maladaptive schemas and social phobia. Cogn Ther Res 2006;30:571-584.

42. Hale WW, Raaijmakers Q, Muris P, van Hoof A, Meeus W. Developmental trajectories of adolescent anxiety disorder symptoms: a 5-year prospective community study. J Am Acad Child Adolesc Psychiatry 2008;47:556-564.

43. Riso LP, Mcbride C. Introduction: A Return to a Focus on Cognitive Schemas. In: Riso LP, du Toit PL, Stein DJ, Young JE, Editors. Cognitive Schemas and Core Beliefs in Psychological Problems: A Scientist-Practitioner Guide. Washington, DC: American Psychological Association; 2007.

44. DiBartolo PM, Albano AM, Barlow DH, Heimberg RG. Cross-informant agreement in the assessment of social phobia in youth. J Abnorm Child Psychol 1998;26:213-220.

45. Dodge KA, Coie JD, Lynam D. Aggression and Antisocial Behavior in Youth. In: Damon W, Lerner RM, Editors. Handbook of Child Psychology: Vol 3 Social, Emotional, and Personality Development. NJ: Wiley: Hoboken; 2006. 